ORIGINAL ARTICLE

\title{
Poor outcome in primary intracerebral haemorrhage: results of a matched comparison
}

\author{
M Barber, G Roditi, D J Stott, P Langhorne
}

Postgrad Med J 2004;80:89-92. doi: 10.1136/pmj.2003.010967

See end of article for authors' affiliations

.....................

Correspondence to: Dr Mark Barber, Academic Section of Geriatric Medicine, Third Floor, Centre Block, Royal Infirmary, 84 Castle Street, Glasgow G4 OSF, UK; M.Barber@

clinmed.gla.ac.uk

Submitted 10 June 2003 Accepted 19 July 2003
Background: Primary intracerebral haemorrhage $(\mathrm{PICH})$ is associated with a poorer outcome than cerebral infarction. This study aimed to determine whether this is explained by the clinical severity of stroke.

Methods: An observational study of outcome in consecutive admissions with acute $\mathrm{PICH}$ and ischaemic stroke was undertaken. A nested case-control analysis, matched on a 1:2 basis for age, pre-stroke disability, early neurological impairment (Scandinavian Stroke Scale; SSS), and Oxfordshire Community Stroke Project classification was then performed. Follow up was at 30 days and at hospital discharge. Results: Overall, 679 subjects were included in the analysis. Of these, 53 (8\%) had PICH; this group had more severe initial neurological impairment (day 3 SSS $28 \vee 45$ points, $p<0.001$ ) and a higher prevalence of total anterior circulation strokes $(55 \% v 21 \%, \mathrm{p}<0.001)$ than did the group admitted with ischaemic strokes. Outcomes were poorer in the $\mathrm{PICH}$ group, with $36 \%$ inpatient mortality and $68 \%$ of survivors having a day 30 modified Rankin Scale (MRS) of at least 3 (compared with 13\% and 52\%, respectively, in the ischaemic stroke group). Following matching for baseline clinical characteristics, the $\mathrm{PICH}$ group had a higher mortality, but this was not statistically significant; the day 30 MRS and institutionalisation rates in survivors were similar in the matched haemorrhage and infarct groups.

Conclusions: Compared with ischaemic stroke, $\mathrm{PICH}$ is associated with higher mortality and increased disability in survivors. The severity of clinical stroke is a major contributor to these poor outcomes; baseline characteristics, however, do not fully explain outcome differences.

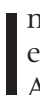
European populations approximately $10 \%$ of stroke events are primary intracerebral haemorrhages (PICH). ${ }^{1-3}$

Although these are a minority of all strokes, they remain a difficult management problem. It is well recognised that PICH patients suffer poor outcomes, ${ }^{134}$ and, as yet, no routine effective acute interventions have been developed to treat this group. Over the last 10 years there has been a significant expansion in the number of hospitals providing stroke care in specialist acute and rehabilitation stroke wards. Evidence suggests that these units improve outcome after acute stroke, $^{5}$ and this is particularly likely to benefit those with PICH.

Our experience has been that although mortality seems high in patients admitted with PICH, many do progress surprisingly well. This seems particularly true if comparisons are made between subjects with similar severity of stroke at onset, especially if subjects avoid complications over the first few days. It has been suggested that PICH has no influence on mortality once the effect of initial stroke severity has been taken into consideration. ${ }^{7}$ Most analyses, however, have not controlled for stroke severity when assessing outcomes in $\mathrm{PICH}$ and acute ischaemic stroke. We, therefore, decided to perform a nested case-control analysis to adjust for stroke severity so that we could compare outcomes in the two groups.

\section{METHODS}

The initial part of our comparison was a univariate analysis assessing outcomes in subjects with PICH (defined as spontaneous intracerebral haemorrhage not attributable to an underlying cause) and ischaemic stroke. Consecutive patients admitted with acute stroke to an urban teaching hospital, over a two year period, were registered in the database. All data were collected prospectively as part of a research protocol. A single experienced stroke radiologist, who had no access to data on patient outcomes, reviewed computed tomograms of all subjects with suspected PICH. Evidence of trauma or suspicion of haemorrhagic transformation of an ischaemic stroke on brain imaging led to exclusion. Subjects were also excluded if they died or were transferred to a neurosurgical unit within three days, or if no Oxfordshire Community Stroke Project (OCSP) classification was available. Patients were excluded if brain imaging was not performed.

Neurological impairment was measured using items of the Scandinavian Stroke Scale (SSS) ${ }^{8}$ at baseline and on day 3 . Modified Rankin Scale (MRS) ${ }^{9}$ scores were recorded on day 3 and day 30. A research nurse, who was unaware of the present analysis, carried out all assessments. Blood pressure was measured non-invasively on admission (Propaq 104EL, Protocol Systems Inc). Mean arterial blood pressure (MABP) was calculated using the formula DBP $+1 / 3$ (SBP-DBP), where DBP is diastolic blood pressure and SBP is systolic blood pressure.

The second part of our investigation consisted of a nested case-control analysis. Subjects with PICH were matched with ischaemic stroke controls using a 1:2 frequency matching technique. Matching was performed using a pivot table in the software package SPSS for Windows version 9 (SPSS Inc, Chicago, Illinois). Subjects were included without outcome results being available using their unique hospital identification number. Cases were matched to controls on the basis of day 3 SSS (0-10, 11-20, 21-30, 31-40, and 41-46), OCSP

Abbreviations: DBP, diastolic blood pressure; LACS, lacunar stroke; $M A B P$, mean arterial blood pressure; MRS, modified Rankin Scale; OCSP, Oxfordshire Community Stroke Project; PACS, partial anterior circulation stroke; PICH, primary intracerebral haemorrhage; POCS, posterior circulation stroke; SBP, systolic blood pressure; SSS, Scandinavian Stroke Scale; TACS, total anterior circulation stroke 
classification (total anterior circulation stroke (TACS), partial anterior circulation stroke (PACS), lacunar stroke (LACS), or posterior circulation stroke (POCS) $),{ }^{10}$ pre-stroke MRS band (0-2 or 3-5), and age band $(20-40,41-60,61-70,71-80,81-$ 90, or 91-100 years) in that order. Matching was performed on the basis of first available matching case within the database. Only after matching was completed were individual patient data included in the final database for group comparison analysis.

Ethical approval for gathering the dataset was obtained from the local research ethics committee.

\section{Statistical analysis}

Comparison between the PICH and ischaemic stroke groups was performed using Mann-Whitney $\mathrm{U}$ tests and unpaired $t$ tests where appropriate. All results are expressed as median (interquartile range) unless otherwise stated. Further analysis of the nested cases and controls was by conditional logistic regression. All statistical analyses were carried out using SPSS.

\section{RESULTS}

Brain imaging was performed before hospital discharge in 769 out of the 873 subjects in our database $(88 \%)$. Nine patients $(1 \%)$ died or were transferred to a neurosurgical unit within three days, and 81 (9\%) had no OCSP classification. After these exclusion criteria were applied, we were left with 679 cases for analysis. The median age was $70(62-79)$ years, and $49 \%$ were female. The median abbreviated SSS score (excluding the gaze, orientation, and gait domains; maximum score of 36) on admission was 29 (20-32). With regard to clinical classification, $24 \%$ had TACS, $39 \%$ had PACS, $28 \%$ had LACS, and $9 \%$ had POCS. The in-hospital mortality was $14 \%$.

After clinical and radiological review, 53 cases (8\%) were classed as PICH. The baseline characteristics of the PICH and infarction groups are shown in table l. Important differences between the two groups include a longer delay to admission ( 4 v 2 hours, $\mathrm{p}<0.05$ ), less serious neurological impairment (abbreviated SSS score of $29 v 23, \mathrm{p}<0.001$ ), fewer TACS $(21 \% v 55 \%, \mathrm{p}<0.001)$, a lower incidence of dysphagia $(22 \% v$ $53 \%, \mathrm{p}<0.001$ ), lower admission MABP (114 mm Hg $v$
$125 \mathrm{~mm} \mathrm{Hg}, \mathrm{p}<0.001)$, and a more frequent past history of stroke $(30 \% \vee 17 \%, \mathrm{p}<0.05)$ in the cerebral infarction group. Outcomes for the PICH and ischaemic stroke groups are shown in table 2. These were worse in the PICH group with respect to mortality, length of stay and functional status (all $\mathrm{p}<0.01$ ). There was a non-significant trend towards an increased need for institutional care in the PICH survivors $(18 \% v 12 \%, \mathrm{p}=0.29)$.

In the unmatched database a logistic regression analysis was performed to examine independent predictors of poor outcome (MRS of at least 3 or death). Independent variables included in the equation were age (odds ratio 1.03 for each year, $p=0.0001$ ), OCSP classification (TACS or other; odds ratio $2.99, \mathrm{p}<0.002$ ), abbreviated SSS on admission (odds ratio $0.91, p<0.0001$ ), dysphagia after admission (odds ratio 2.01, $\mathrm{p}<0.05$ ), MABP (odds ratio 0.99, $\mathrm{p}=0.12$ ), pre-stroke MRS of at least 3 (odds ratio 3.45, p $<0.0001$ ), history of diabetes (odds ratio 0.98, $\mathrm{p}=0.82$ ), and previous stroke (odds ratio $1.25, \mathrm{p}=0.08$ ). This supported our predefined matching criteria for the nested case-control analysis.

Following matching for day 3 neurological impairment, OCSP clinical classification, pre-stroke function and age, the only significant baseline imbalance between the PICH group and the infarction group was in admission MABP (125 v $116 \mathrm{~mm} \mathrm{Hg}, \mathrm{p}<0.01$ ) (see table 1 ). Admission heart rates were very similar ( $81 \vee 84$ beats/min, $\mathrm{p}=0.96$ ). Although there remained non-significant trends towards increased mortality and length of stay, the magnitudes of these differences were greatly reduced when compared with the unmatched analysis (see table 2). Conditional logistic regression of the matched subjects, taking into account the pairing of the dataset, provided an odds ratio of 1.94 (95\% confidence interval 0.67 to $5.63, p=0.22$ ) for poor outcome in the PICH group. If MABP is added to this model, we find that PICH remains non-significantly associated with poor outcome, while MABP provides an odds ratio of 1.03 (95\% confidence interval 1.01 to $1.05, \mathrm{p}<0.01$ ) for each rise of 1 $\mathrm{mm} \mathrm{Hg}$.

\section{DISCUSSION}

We have confirmed that, in an unselected consecutive series of acute stroke admissions to an urban teaching hospital,

Table 1 Comparison of baseline data between PICH, total ischaemic stroke, and matched ischaemic stroke groups. Abbreviated SSS is made up of all components except gaze, orientation, and gait scores (giving a possible maximum of 36 points). For continuous data results are expressed as median (interquartile range) unless otherwise stated

\begin{tabular}{|c|c|c|c|}
\hline & $\mathrm{PICH}(n=53)$ & $\begin{array}{l}\text { Cerebral infarction } \\
(n=626)\end{array}$ & $\begin{array}{l}\text { Matched cerebral } \\
\text { infarctions }(n=106)\end{array}$ \\
\hline Age (years) & $74(62-80)$ & $70(62-79)$ & $71(63-78)$ \\
\hline Sex (\% female) & 45.3 & 49.5 & 41.5 \\
\hline Time from symptoms to admission (hours) & $2(1-6)$ & $4(1-24)^{*}$ & $2(1-13)$ \\
\hline Baseline abbreviated SSS score & $23(10-30)$ & $29(21-32)^{\star * *}$ & $20(9-29)$ \\
\hline Day 3 total SSS score & $28(6-46)$ & $45(31-54)^{* * *}$ & $30(10-45)$ \\
\hline Reduced conscious level (\%) & $16(30.2)$ & $55(8.8)^{\star \star \star}$ & $26(24.5)$ \\
\hline \multicolumn{4}{|l|}{ OCSP classification (\%) } \\
\hline TACS & $29(54.7)$ & $134(21.4)^{\star \star \star}$ & $56(52.8)$ \\
\hline PACS & $13(24.5)$ & $253(40.4)$ & $28(26.4)$ \\
\hline LACS & $5(9.4)$ & $186(29.7)$ & $10(9.4)$ \\
\hline POCS & $6(11.3)$ & $53(8.5)$ & $12(11.3)$ \\
\hline Dysphagia after admission (\%) & $28(52.8)$ & $137(21.9)^{\star \star \star}$ & $51(48.1)$ \\
\hline MABP on admission $(\mathrm{mm} \mathrm{Hg})$ & $125(112-147)$ & $114(100-128)^{\star * *}$ & $116(104-129)^{*}$ \\
\hline Incontinence at day $3(\%)$ & $37(69.8)$ & $208(33.2)^{\star \star \star}$ & $58(54.7)$ \\
\hline Pre-stroke MRS band 3-5 (\%) & 7 (13.2) & $110(17.6)$ & $13(12.3)$ \\
\hline Past history of diabetes (\%) & $8(15.1 \%)$ & $102(16.3)$ & $13(12.3)$ \\
\hline Previous stroke (\%) & $9(17.0)$ & $190(30.4)^{*}$ & $27(25.5)$ \\
\hline Previous hypertension (\%) & $19(35.8)$ & $312(49.8)$ & $50(47.2)$ \\
\hline
\end{tabular}


Table 2 Outcome comparisons between PICH, total ischaemic stroke, and matched ischaemic stroke groups. For continuous data results are expressed as median (interquartile range) unless otherwise stated

\begin{tabular}{|c|c|c|c|}
\hline & $\mathrm{PICH}(n=53)$ & $\begin{array}{l}\text { Cerebral infarction } \\
(n=626)\end{array}$ & $\begin{array}{l}\text { Matched cerebral } \\
\text { infarctions }(n=106)\end{array}$ \\
\hline $\begin{array}{l}\text { Dead at } 30 \text { days }(\%) \\
\text { Death as discharge diagnosis (\%) } \\
\text { Poor outcome at } 30 \text { days (dead or } \\
\text { MRS of at least } 3 \text { ) (\%) }\end{array}$ & $\begin{array}{l}15(28.3) \\
19(35.8) \\
42(79.2)\end{array}$ & $\begin{array}{l}43(6.9)^{* * *} \\
79(12.6)^{* * *} \\
363(58.0)^{* *}\end{array}$ & $\begin{array}{l}19(17.9) \\
28(26.4) \\
77(72.6)\end{array}$ \\
\hline $\begin{array}{l}\text { Survivors to discharge } \\
30 \text { day MRS of at least } 3(\%) \\
30 \text { day Barthel index } \\
\text { Days to discharge } \\
\text { Institutional care (\%) }\end{array}$ & $\begin{array}{l}34 \\
23(67.6) \\
8(3-18) \\
47(11-101) \\
6(17.6)\end{array}$ & $\begin{array}{l}547 \\
282(51.6) \\
16(10-19)^{\star * *} \\
16(8-41)^{* *} \\
63(11.5)\end{array}$ & $\begin{array}{l}78 \\
49(62.8) \\
14(4-19) \\
24(12-65) \\
13(16.7)\end{array}$ \\
\hline
\end{tabular}

patients with PICH have higher mortality and worse functional outcomes than patients with acute ischaemic stroke. Outcome is very poor in this group, with $47 \%$ of subjects dying or being institutionalised compared with $23 \%$ of unselected ischaemic stroke admissions. Subjects with PICH suffer more severe strokes with a higher prevalence of TACS and more severe neurological impairment as measured by the SSS. Once these factors, along with age, are controlled for in a nested analysis, the differences between the groups are much reduced, suggesting that clinical stroke severity is an important factor in poor outcome in PICH. There remains, however, a non-significant trend towards poorer outcome in PICH even after matching.

We chose day 3 severity of neurological impairment as a matching variable to reduce the effects of very early death and deterioration in the PICH group. Before commencing the analysis, we decided to match our cases to controls on the basis of day 3 SSS score, OCSP classification, pre-stroke MRS, and age. This was supported by a multivariate analysis of the unmatched dataset. Matching using these variables has removed some of the effect of PICH on poor outcome but not all. The unadjusted odds ratio for poor outcome in the PICH group was 1.94 (95\% confidence interval 0.67 to 5.63 ) in the nested matched dataset. This compares with an odds ratio of 2.77 (95\% confidence interval 1.40 to 5.47 ) in the unmatched database. Much of the "poor outcome" is accounted for by in-hospital deaths. In those who survived to discharge, outcomes were similar in the PICH and infarction groups in terms of MRS at 30 days and the need for institutionalisation.

Important independent predictors of 30 day mortality in patients with PICH include initial level of consciousness and haematoma volume. ${ }^{11-13}$ In performing our matching we achieved a reasonable match for numbers of subjects with reduced consciousness on admission (30\% for PICH $v 25 \%$ for ischaemic stroke, $p=0.445$ ). If computed tomography had been performed at similar times then reporting infarct or haemorrhage volume would have been useful in our analysis. However, computed tomography was performed as part of routine clinical practice rather than in a formal research protocol, and, therefore, there was a wide variation in delays to imaging (interquartile range $1-5$ days). Because of this variation, patients' infarctions and haemorrhages will have been at different stages of evolution, and measurement of lesion size is unlikely to be informative. This is emphasised by the fact that many subjects in the cerebral infarction group (27\% of the total infarct group, $19 \%$ of the matched infarct group) had no visible lesion when scanned. Brain imaging was accordingly used only to classify strokes as haemorrhages or infarctions, particularly as we were mainly interested in early clinical predictors.
Franke et al found that pineal gland displacement on computed tomography, admission blood glucose, Glasgow Coma Scale eye and motor scores, and haematoma volume predicted 2 day mortality in a hospital cohort admitted during the late $1980 \mathrm{~s} .{ }^{14}$ They performed a similar nested casecontrol analysis to ours on day 2 survivors, matched primarily on the basis of MRS with the closest possible eye and motor scores of the Glasgow Coma Scale and age. In contrast to our results, they found identical outcomes in the matched dataset without even trends towards poor outcome in the $\mathrm{PICH}$ group. This discrepancy may be due to their choice of MRS as the main matching variable.

We found higher MABP in the PICH group. Blood pressure is normally initially elevated after acute stroke but falls over the next week in acute cerebral infarction and PICH. ${ }^{15-18}$ High SBP on admission predicts early neurological deterioration after cerebral infarction and subsequent poor outcome. ${ }^{18} 19$ PICH patients are known to have higher SBP and DBP on admission than ischaemic stroke patients. ${ }^{16}{ }^{18} 20$ These differences in blood pressure remain significant after correction for stroke severity; age, however, may be an important factor. ${ }^{15} 20$ Even after matching there remained a non-significant, but potentially important, difference in age between our groups. Carlberg et al previously found that high blood pressure in PICH subjects was not predictive of death at 30 days (apart from in a subgroup with impaired consciousness). ${ }^{21}$ In our unmatched dataset we found no significant correlation between MABP and poor outcome after correction for age, stroke severity, and pre-morbid conditions in a multivariate analysis. Even though we found that MABP is associated with poor outcome, it seems unlikely that this alone is the cause of worse prognosis in the PICH group; it may reflect some other unrecognised process.

Our 30 day case fatality rates for PICH and infarction tended to be lower ( $28 \%$ and $7 \%$, respectively) than in previous population studies. ${ }^{1}{ }^{4}$ The hospital based approach of our study means that some subjects died before hospital admission and some subjects with milder strokes were managed as outpatients. Hospital based studies of PICH have shown similar 30 day case fatality rates to ours. ${ }^{1122}$ In our study, $12 \%$ of patients had no brain imaging (or autopsy if they died before imaging). Case fatality at 30 days in this group was $27 \%$, which is again lower than in population based studies. In some cases the reason for this lack of imaging may be that subjects had only minor symptoms and were discharged to outpatient follow up and investigation. Other subjects may have been so unwell or have had such poor pre-morbid status that imaging was felt to be clinically inappropriate. It is not possible clinically to diagnose PICH reliably, ${ }^{23}$ and it was, therefore, necessary to exclude these non-imaged subjects. We feel that this is unlikely to have 
significantly biased our results. At 30 days our period of assessment was relatively short. This decision was taken as we believed that a longer period might lead to incomplete follow up data. Time to discharge in the PICH group was longer (47 v 24 days), and this may reflect a delayed recovery in the PICH group, particularly as final discharge destinations were similar.

We have confirmed, in an unselected consecutive group of acute stroke admissions, that inpatient mortality after PICH is high and that survivors are more likely to be dependent. Mortality remains higher (but no longer statistically significantly so) in the PICH group after matching for stroke severity, age, and previous dependency. Dependency levels at day 30 and the need for institutionalisation in those who survive to hospital discharge, however, are similar in the $\mathrm{PICH}$ and matched infarct groups. It is important to measure clinical stroke severity, and we recommend the routine use of standardised assessment tools such as the SSS or the modified National Institutes of Health stroke scale ${ }^{24}$ in acute stroke admissions to provide prognostic information in PICH and ischaemic stroke.

\section{ACKNOWLEDGEMENTS}

MB is an NHS Education for Scotland/Chief Scientist Office Clinical Research Fellow. Chest, Heart and Stroke Scotland and Glaxo Wellcome supported the hospital stroke register.

\section{Authors' affiliations}

M Barber, D J Stott, P Langhorne, Academic Section of Geriatric Medicine, Royal Infirmary, Glasgow, UK

G Roditi, Department of Radiology, Royal Infirmary, Glasgow, UK

\section{REFERENCES}

1 Hollander M, Koudstaal PJ, Bots ML, et al. Incidence, risk, and case fatality of first ever stroke in the elderly population. The Rotterdam Study. J Neurol Neurosurg Psychiatry 2003;74:317-21.

2 Wolfe CDA, Giroud M, Kolominsky-Rabas P, et al. Variations in stroke incidence and survival in three areas of Europe. Stroke 2000;31:2074-9.

3 Bamford J, Sandercock P, Dennis M, et al. A prospective study of acute cerebrovascular disease in the community: the Oxfordshire Community Stroke Project-1981-86. 2. Incidence, case fatality rates and overall outcome at one year of cerebral infarction, primary intracerebral and subarachnoid haemorrhage. J Neurol Neurosurg Psychiatry 1990;53:16-22.
4 Thrift AG, Dewey HM, Macdonell RAL, et al. Incidence of the major stroke subtypes: initial findings from the North East Melbourne Stroke Incidence Study (NEMESIS). Stroke 2001;32:1732-8.

5 Stroke Units Trialists' Collaboration. Organised inpatient (stroke unit) care for stroke (Cochrane review). The Cochrane library. Chichester: John Wiley, issue 4, 2003.

6 Ronning OM, Guldvog B, Stavem K. The benefit of an acute stroke unit in patients with intracranial haemorrhage: a controlled trial. J Neurol Neurosurg Psychiatry 2001;70:631-4.

7 Jorgensen HS, Nakayama $\mathrm{H}$, Raaschou $\mathrm{HO}$, et al. Intracerebral hemorrhage versus infarction: stroke severity, risk factors, and prognosis. Ann Neurol 1995;38:45-50.

8 Scandinavian Stroke Study Group. Multicenter trial of hemodilution in ischaemic stroke-background and study protocol. Stroke 1985;16:885-90.

9 Van Swieten JC, Koudstaal PJ, Visser MC, et al. Interobserver agreement for the assessment of handicap in stroke patients. Stroke 1988;19:604-7.

10 Bamford J, Sandercock P, Dennis M, et al. Classification and natural history of clinically identifiable subtypes of cerebral infarction. Lancet 1991;337:1521-6.

11 Nilsson OG, Lindgren A, Brandt L, et al. Prediction of death in patients with primary intracerebral hemorrhage: a prospective study of a defined population. J Neurosurg 2002;97:531-6.

12 Broderick JP, Brott TG, Duldner JE, et al. Volume of intracerebral hemorrhage. A powerful and easy-to-use predictor of 30-day mortality. Stroke 1993;24:987-93.

13 Hemphill JC III, Bonovich DC, Besmertis L, et al. The ICH score: a simple reliable grading scale for intracerebral hemorrhage. Stroke 2001;32:891-7.

14 Franke CL, Van Swieten JC, Algra A, et al. Prognostic factors in patients with intracerebral haematoma. J Neurol Neurosurg Psychiatry 1992;55:653-7.

15 Morfis L, Schwartz RS, Poulos R, et al. Blood pressure changes in acute cerebral infarction and haemorrhage. Stroke 1997;28:1401-5.

16 Harper G, Castleden C, Potter J. Factors affecting changes in blood pressure after acute stroke. Stroke 1994;25:1726-9.

17 Britton M, Carlsson A. Very high blood pressure in acute stroke. J Intern Med 1990;228:611-15.

18 Robinson T, Waddington A, Ward-Close S, et al. The predictive role of 24hour compared to casual blood pressure levels on outcome following acute stroke. Cerebrovasc Dis 1997;7:264-72.

19 Dávalos A, Cendra E, Tereul J, et al. Deteriorating ischemic stroke: risk factors and prognosis. Neurology 1990;40:1865-9.

20 Jørgensen $\mathbf{H}$, Nakayama $\mathrm{H}$, Christensen $\mathrm{H}$, et al. Blood pressure in acute stroke. Cerebrovasc Dis 2002;13:204-9.

21 Carlberg B, Asplund K, Hägg E. The prognostic value of admission blood pressure in patients with acute stroke. Stroke 1993;24:1372-5.

22 Fieschi C, Carolei A, Fiorelli M, et al. Changing prognosis of primary intracerebral hemorrhage: results of a clinical and computed tomographic follow-up study of 104 patients. Stroke 1988;19:192-5.

23 Besson G, Robert C, Hommel M, et al. Is it clinically possible to distinguish nonhemorrhagic infarct from hemorrhagic stroke. Stroke 1995;26:1205-9.

24 Meyer BC, Hemmen TM, Jackson CM, et al. Modified National Institutes of Health stroke scale for use in stroke clinical trials: prospective reliability and validity. Stroke 2002;33:1261-6. 